\title{
Structure Effect of Diacid/Triacid on Self-Assembly of Binary Organogels Based on Glutamic Acid Amino Derivative
}

\author{
Jinming Dai, Wei Hong, and Youbo Di \\ College of Textile Engineering and Art, Taiyuan University of Technology, Taiyuan 030024, China \\ Correspondence should be addressed to Jinming Dai; jinmingdai12@126.com and Youbo Di; youbodi123@126.com
}

Received 10 June 2013; Accepted 4 July 2013

Academic Editor: Xinqing Chen

Copyright (C) 2013 Jinming Dai et al. This is an open access article distributed under the Creative Commons Attribution License, which permits unrestricted use, distribution, and reproduction in any medium, provided the original work is properly cited.

\begin{abstract}
The gelation behaviors of binary organogels composed of N-(4-aminobenzoyl)-L-glutamic acid diethyl ester with sebacic acid and citric acid in various organic solvents were designed and investigated. Their gelation behaviors in 20 solvents were tested as new binary organic gelators. It showed that the molecular structures and organic solvents have played a crucial role in the gelation behavior of all gelator mixtures. More carboxyl groups in molecular skeletons in the present mixture gelators are unfavorable for the gelation of organic solvents. The mixture containing sebacic acid can form 5 kinds of organogels, while another mixture containing citric acid can only form 3 kinds of organogels in different solvents. Morphological studies revealed that the gelator molecules self-assemble into different aggregates from wrinkle and belt to fiber with change of solvents. Spectral studies indicated that there existed different $\mathrm{H}$-bond formations and hydrophobic forces, depending on solvents and molecular structures. The as-prepared nanomaterials have wide perspectives in nanoscience and functional textile materials with special microstructures.
\end{abstract}

\section{Introduction}

In recent years, organized organogels have become one of the hot areas in soft matter research due to their scientific values and many potential applications in biomedical field, including tissue engineering, controlled drug release, and medical implants $[1-8]$. The gels are usually considered as supramolecular gels, in which the gelator molecules selfassemble into three-dimensional networks in which the solvent is trapped via various noncovalent interactions, such as hydrogen bonding, $\pi-\pi$ stacking, van der Waals interaction, dipole-dipole interaction, coordination, solvophobic interaction, and host-guest interaction [9-12].

In addition, $\mathrm{L}$-glutamic acid is one of the essential amino acids in human bodies and has many hydrogen-bonding sites, which is usually necessary to regulate the assembling modes $[13,14]$. In some reported work, the gelation properties of some cholesterol imide derivatives consisting of cholesteryl units and photoresponsive azobenzene substituent groups have been investigated [15]. They found that a subtle change in the head group of azobenzene segment can produce a dramatic change in the gelation behavior of both compounds. In addition, the gelation properties of bolaform and trigonal cholesteryl derivatives with different aromatic spacers have been characterized [16].

As an interesting work, herein, we have designed and prepared new binary organogels composed of $\mathrm{N}$-(4aminobenzoyl)-L-glutamic acid diethyl ester with sebacic acid and citric acid, respectively. For present diacid/triacid, the number of carboxyl group in molecular skeletons shifted from 2 to 3 , respectively. We have found that both present mixtures could form different organogels in various organic solvents. Morphological and spectral characterization of the organogels revealed different structures of the aggregates in the gels. We have investigated the structure effect of diacid/triacid and solvents on the nanostructures of these organogels.

\section{Experiments}

2.1. Materials. The starting materials, $\mathrm{N}-(4$-aminobenzoyl)L-glutamic acid diethyl ester, sebacic acid, and citric acid, were purchased from Alfa Aesar Tianjin Chemicals, Aldrich Chemicals, and TCI Shanghai Chemicals, respectively. Other used reagents were all for analysis purity from Beijing Chemicals and were distilled before use. 


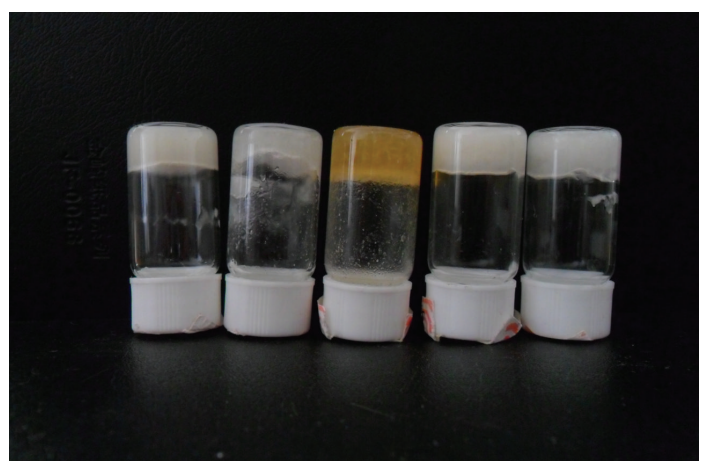

(a)

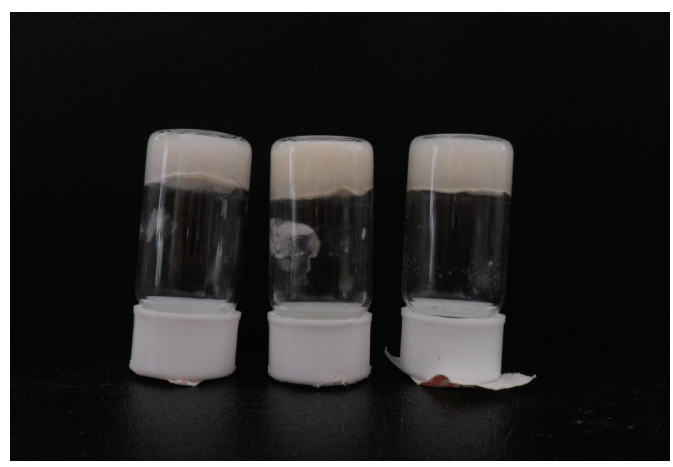

(b)

FIGURE 1: Photographs of the as-prepared organogels of Glu-SA (a) and Glu-CA (b) in different solvents, respectively.

2.2. Gelation Test. All mixed organogels were prepared according to a simple procedure. Firstly, both acid and N(4-aminobenzoyl)-L-glutamic acid diethyl ester were mixed with molecular molar ratios of $1: 2$ or $1: 3$ according to the number matching intermolecular carboxylic acid and amine group, respectively. Then, a weighted amount of binary mixtures and a measured volume of selected pure organic solvent were placed into a sealed glass bottle, and the solution was heated in a water bath until the solid was dissolved. Then, the solution was cooled to room temperature in air, and the test bottle was inversed to see if a gel was formed [17]. When the binary mixtures formed a gel by immobilizing the solvent at this stage, it was denoted by "G." For the systems in which only solution remained until the end of the tests, they were referred to as solution (S). When the binary mixtures were formed into a few precipitate in some solvent, they was denoted as a "PS."

2.3. Measurements. Firstly, the xerogel was prepared by a vacuum pump for more than $24 \mathrm{~h}$. The dried sample thus obtained was attached to copper foil, glass, and $\mathrm{CaF}_{2}$ slice for morphological and spectral investigation, respectively. Before SEM measurement, the samples were coated on copper foil fixed by conductive adhesive tape and shielded by gold. SEM pictures of the xerogel were taken on a Hitachi S- 4800 field emission scanning electron microscopy with the accelerating voltage of 5-15 kV. Transmission FT-IR spectra of the xerogel were obtained by Nicolet iS/10 FT-IR spectrophotometer from Thermo Fisher Scientific Inc. by averaging 32 scans and at a resolution of $4 \mathrm{~cm}^{-1}$. The XRD measurement was conducted using a Rigaku D/max 2550PC diffractometer (Rigaku Inc., Tokyo, Japan). The XRD pattern was obtained using $\mathrm{CuK} \alpha$ radiation with an incident wavelength of $0.1542 \mathrm{~nm}$ under a voltage of $40 \mathrm{kV}$ and a current of $200 \mathrm{~mA}$. The scan rate was $0.5^{\circ} / \mathrm{min}$.

\section{Results and Discussions}

3.1. Gelation Behaviors. Firstly, the gelation performances of both binary mixtures in 20 solvents are tested. The experimental data showed that both binary mixtures could form organogels in special organic solvents, as listed in
TABLE 1: Gelation behaviors of these binary organogels.

\begin{tabular}{lcc}
\hline Solvents & Glu-SA & Glu-CA \\
\hline Acetone & PS & PS \\
Aniline & S & S \\
n-Hexane & PS & PS \\
Toluene & S & S \\
Pyridine & S & S \\
Isopropanol & G & G \\
Cyclopentanone & S & S \\
Cyclohexanone & S & S \\
Nitrobenzene & G & S \\
n-Butanol & G & G \\
Ethanolamine & S & S \\
n-Butyl acrylate & G & PS \\
1,4-Dioxane & S & S \\
Petroleum ether & PS & PS \\
Ethyl acetate & PS & PS \\
Dichloromethane & PS & PS \\
THF & S & S \\
DMF & S & S \\
DMSO & S & S \\
Benzene & G & G \\
\hline soltion; patily & &
\end{tabular}

S: solution; PS: partially soluble; G: gel.

Table 1. The binary mixtures of $\mathrm{N}$-(4-aminobenzoyl)-Lglutamic acid diethyl ester with sebacic acid and citric acid are denoted by Glu-SA and Glu-CA, respectively. For the case of Glu-SA, the mixture can form 5 kinds of organogels in present solvents, including isopropanol, nitrobenzene, n-butanol, n-butyl acrylate, and benzene, respectively. In addition, as for Glu-CA, 3 kinds of organogels were prepared in isopropanol, n-butanol, and benzene, respectively. The photographs of the as-prepared organogels in different solvents were shown in Figure 1. The present data indicated that structure change of diacid/triacid in gelator mixtures can has a profound effect upon the gelation abilities of these studied mixtures. It seemed that more carboxyl groups in molecular skeletons in present mixture gelators are unfavorable for the gelation of organic 


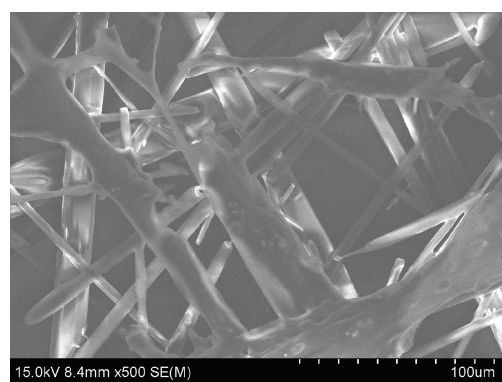

(a)

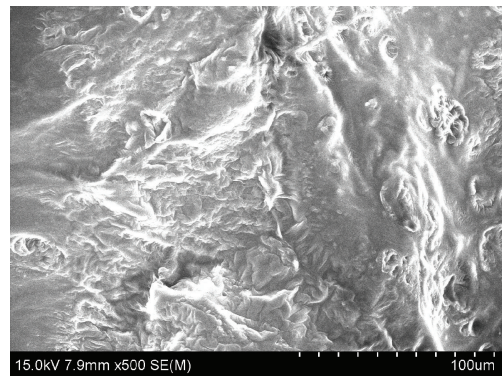

(d)

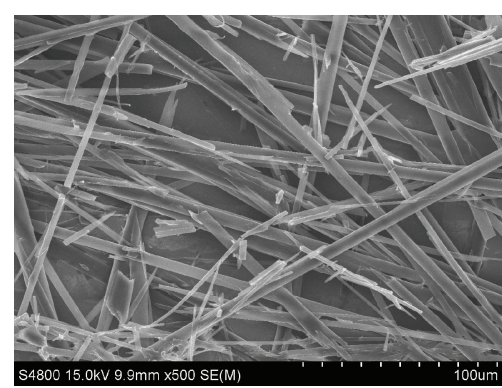

(b)

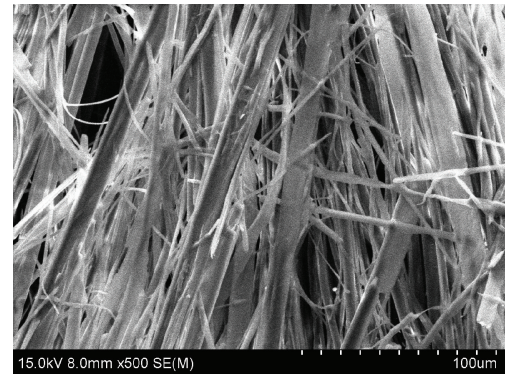

(e)

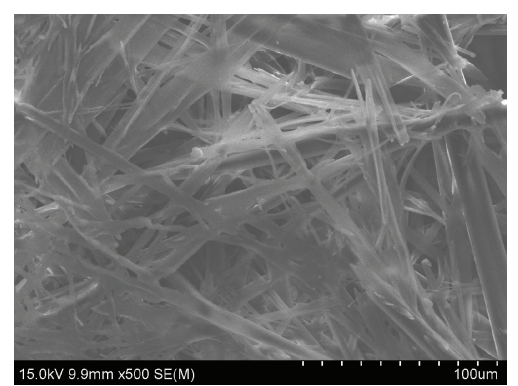

(c)

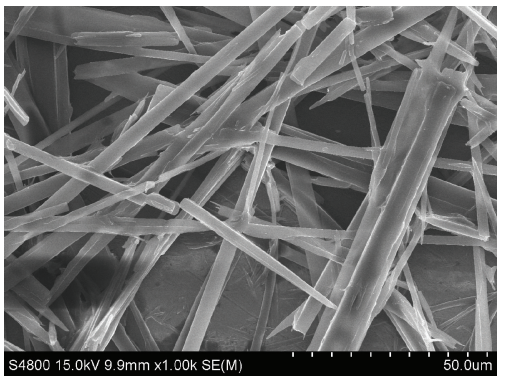

(f)

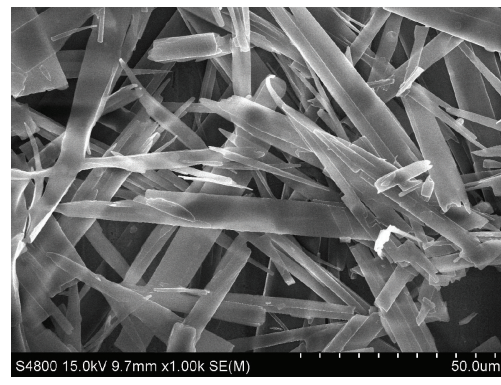

(g)

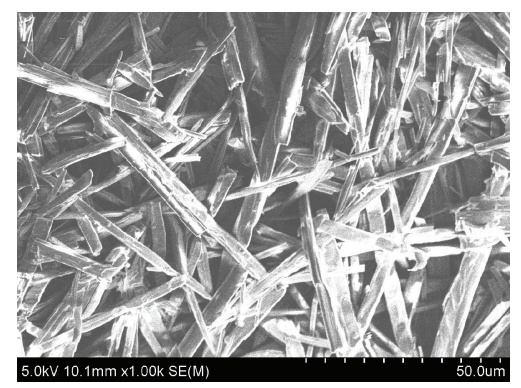

(h)

FIGURE 2: SEM images of xerogels of Glu-SA ((a)-(e): isopropanol, nitrobenzene, n-butanol, n-butyl acrylate, and benzene, resp.) and Glu-CA ((f)-(h): isopropanol, n-butanol, and benzene, resp.).

solvents. In addition, the effect of alkyl chains in Glu-SA for intermolecular stacking in the gel formation process seemed also obvious for all cases. The reason for the variation on the gelation behaviors can be assigned to the change of spatial stacking and self-assembly mode of the gelators due to intermolecular hydrogen bonding of the gelators $[18,19]$, which may shift the ability of the gelator molecules to selfassemble into ordered structures, a necessity for forming organized assembly structures.

3.2. Morphological Investigation. It has been reported that some organized nanoscale superstructures, such as nanofibers, nanoribbons, and nanosheets, could be formed in a supramolecular gel [20]. To obtain a visual insight into nanostructures of present organogels, the typical structures of these gels were studied by SEM technique, as shown in Figure 2. From the present diverse images, it can be obviously investigated that the nanostructures of the xerogels of both mixtures in different solvents are different from each other, and the morphologies of the aggregates change from belt, wrinkle to fiber with change of solvents. In addition, from the images, it is interesting to note that these big belt aggregates were composed of many little fiber-like nanostructures by stacking of the present gelator mixtures. The morphologies of the aggregates shown in SEM images may be rationalized by considering a commonly accepted idea that highly directional intermolecular interactions, such as hydrogen bonding or $\pi-\pi$ interactions, favor formation of organized belt or fiber micro-/nanostructures [21]. The difference of morphologies between different gelator mixtures can be mainly due to the different assembly modes originated from various intermolecular forces, which have played an important role in regulating the intermolecular orderly staking and formation of special aggregates.

3.3. Spectral Investigation. In addition, in order to further investigate the orderly stacking of xerogels nanostructures, XRD of all xerogel from gels was measured, as shown in Figure 3. The curve of Glu-SA xerogel from isopropanol shows main peaks in the angle region ( $2 \theta$ values, $4.84,9.60$, 


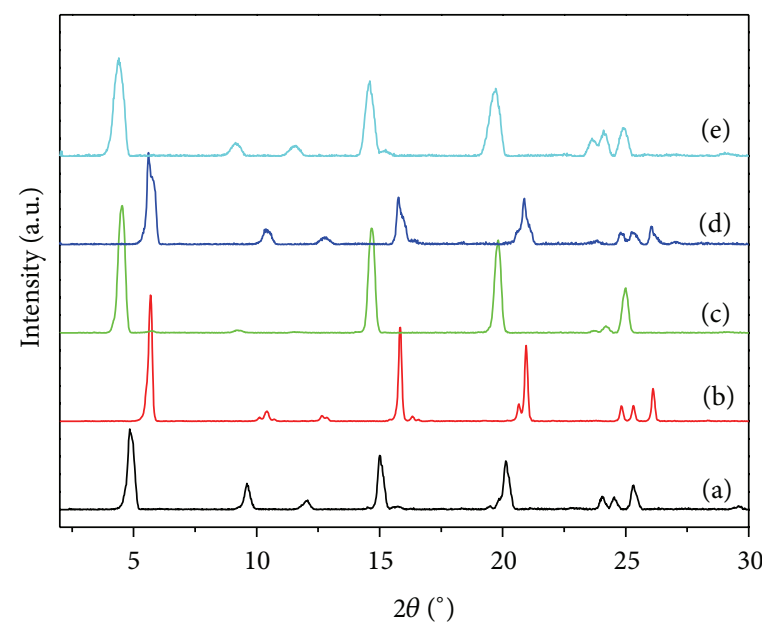

(a)

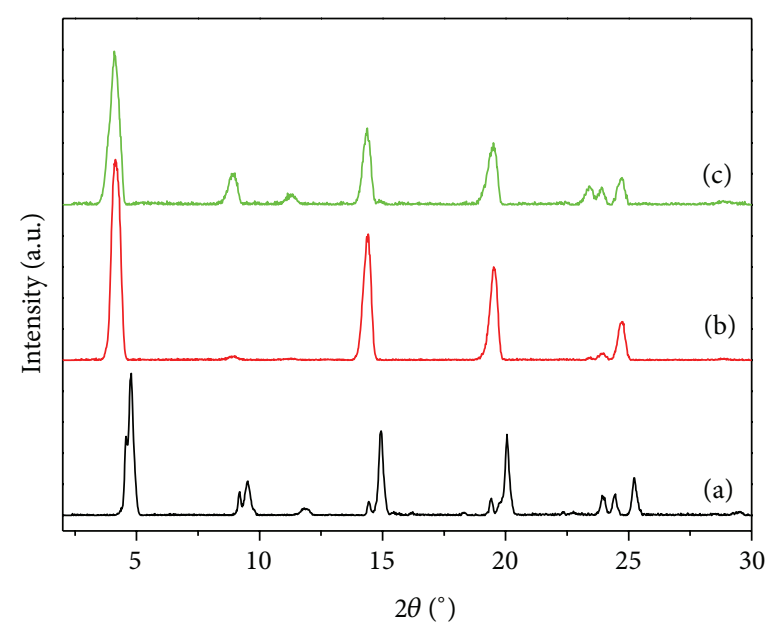

(b)

Figure 3: X-ray diffraction patterns of xerogels: (a) Glu-SA ((a)-(e): isopropanol, nitrobenzene, n-butanol, n-butyl acrylate, and benzene, resp.); (b) Glu-CA ((a)-(c): isopropanol, n-butanol, and benzene, resp.).

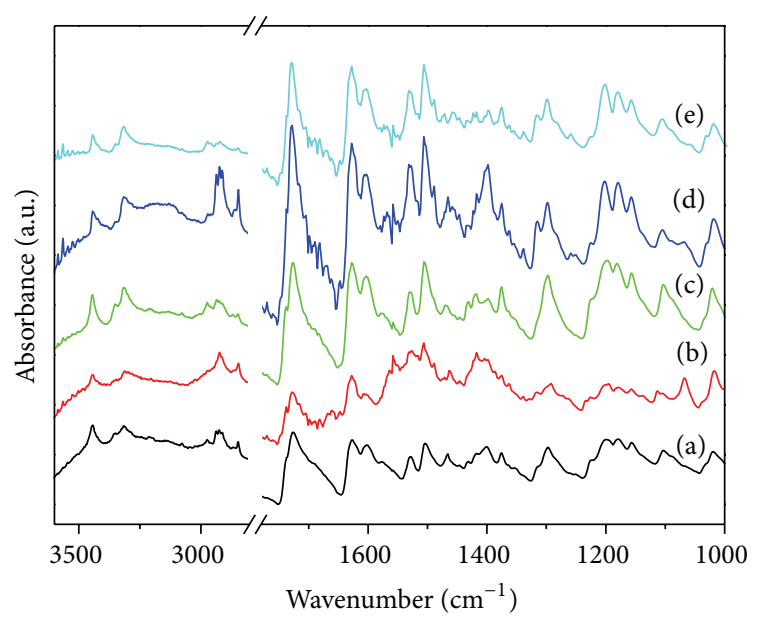

(a)



(b)

FIGURE 4: FT-IR spectra of xerogels: (a) Glu-SA ((a)-(e): isopropanol, nitrobenzene, n-butanol, n-butyl acrylate, and benzene, resp.); (b) Glu-CA ((a)-(c): isopropanol, n-butanol, and benzene, resp.).

$15.02,20.12$, and $25.30^{\circ}$ ) corresponding to $d$ values of 1.83 , $0.92,0.59,0.44$, and $0.35 \mathrm{~nm}$, respectively. The corresponding $d$ values almost follow a ratio of $1: 1 / 2: 1 / 3: 1 / 4: 1 / 5$, suggesting a lamellar structure within the gel with an interlayer distance of $1.83 \mathrm{~nm}$ [22]. The similar curves of Glu-SA xerogels from $\mathrm{n}$-butanol and benzene showed minimum peaks at $2 \theta$ values of 4.54 and $4.38^{\circ}$, respectively, suggesting a little change compared with that from isopropanol mentioned above. However, for the curves of Glu-SA xerogels from nitrobenzene and n-butyl acrylate, the same minimum peaks shifted to $2 \theta$ values of $5.68^{\circ}$, corresponding to $d$ value of $1.56 \mathrm{~nm}$. In addition, for the cases of Glu-CA xerogels from isopropanol, n-butanol, and benzene, the minimum peaks were obtained at $2 \theta$ values of $4.78,4.18$, and $4.10^{\circ}$, corresponding to $d$ values of $1.85,2.12$, and $2.16 \mathrm{~nm}$. The present results described above demonstrated that the many factors had great effect on the self-assembly modes of these gelator mixtures.

It has been widely reported that hydrogen bonding played an important role in the self-assembly process of organogels $[23,24]$. At present, in order to further clarify this and investigate the effect of various factors on self-assembly, the FT-IR spectra of all xerogels of both gelator mixtures were measured, as shown in Figure 4. As far as the spectra of Glu-SA xerogels from different solvents, typical peaks were observed at 3444, 3313,2922, 2851, 1726, 1624, and $1466 \mathrm{~cm}^{-1}$, respectively, which can be assigned to the $\mathrm{N}-\mathrm{H}$ stretching, methylene stretching, carbonyl stretching, amide I band, and methylene scissoring, respectively [25, 26]. These bands indicate $\mathrm{H}$-bond formation between intermolecular amide and carboxylic acid groups in the gel state. In addition, similar spectra were obtained from Glu-CA xerogels 


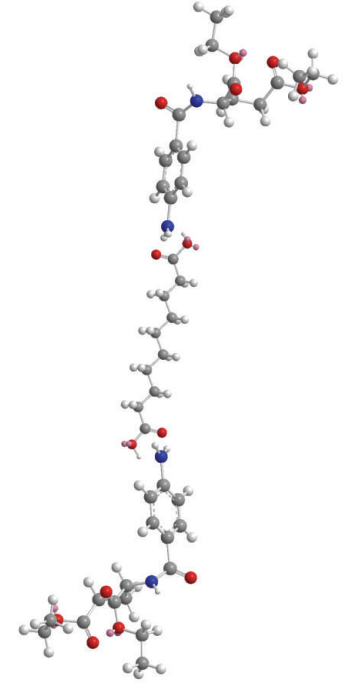

(a) Glu-SA

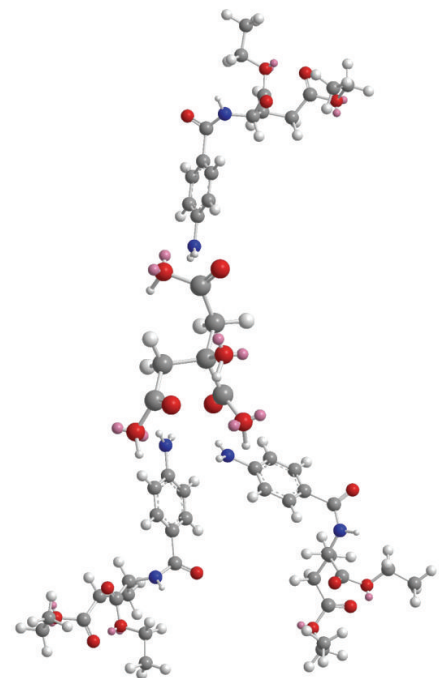

(b) Glu-CA

Figure 5: Possible self-assembly modes of Glu-SA (a) and Glu-CA (b) in organogels.

from isopropanol, n-butanol, and benzene, respectively. This implied that there were differences in the strength of the intermolecular hydrogen bond interactions in these xerogels.

Considering the XRD results described above and the hydrogen-bonding nature of the organized packing of both binary mixtures by FT-IR measurements, the possible selfassembly modes of Glu-SA and Glu-CA in organogels were proposed and schematically shown in Figure 5. The calculated repeating unit of about $1.8 \mathrm{~nm}$ was obtained, which was in well accordance with the obtained experimental values. The present data demonstrated that the structure change of diacid/triacid in gelator mixtures had a great effect on the selfassembly modes and stacking units of the binary gelators.

\section{Conclusion}

The gelation behaviors of binary mixtures of N-(4-aminobenzoyl)-L-glutamic acid diethyl ester with sebacic acid and citric acid in various organic solvents were investigated. The experimental results indicated that the gelation behaviors in different solvents can be regulated by changing molecular structures and organic solvents. The mixture of Glu-SA can form 5 kinds of organogels, while Glu-CA can only form 3 kinds of organogels in different solvents. Morphological studies revealed that the gelator molecules self-assemble into different aggregates from wrinkle and belt to fiber with change of solvents. Spectral studies indicated that there existed different $\mathrm{H}$-bond formations and hydrophobic forces, depending on solvents and molecular structures. The as-prepared nanomaterials have wide perspectives in nanoscience and material fields. The present research work may also give some insight into design and character of new organogelators and functional textile materials with special microstructures.

\section{Conflict of Interests}

The authors declare that they have no any direct financial relation with the commercial identities mentioned in this paper that might lead to a conflict of interest for any of the authors.

\section{Acknowledgments}

This work was supported by the National Natural Science Foundation of China (50943027) and the Foreign Trade and Economic Development Funds of National Development and Reform Commission of China (2150704).

\section{References}

[1] A. R. Hirst, D. K. Smith, and J. P. Harrington, "Unique nanoscale morphologies underpinning organic gel-phase materials," Chemistry, vol. 11, no. 22, pp. 6552-6559, 2005.

[2] F. Xin, H. Zhang, B. Hao et al., "Controllable transformation from sensitive and reversible heat-set organogel to stable gel induced by sodium acetate," Colloids and Surfaces A, vol. 410, pp. 18-22, 2012.

[3] F. S. Schoonbeek, J. H. VanEsch, R. Hulst, R. M. Kellogg, and B. L. Feringa, "Geminal Bis-ureas as gelators for organic solvents: gelation properties and structural studies in solution and in the gel state," Chemistry, vol. 6, no. 14, pp. 2633-2643, 2000.

[4] M. Moniruzzaman and P. R. Sundararajan, "Low molecular weight organogels based on long-chain carbamates," Langmuir, vol. 21, no. 9, pp. 3802-3807, 2005.

[5] B. Escuder, S. Martí, and J. F. Miravet, "Organogel formation by coaggregation of adaptable amidocarbamates and their tetraamide analogues," Langmuir, vol. 21, no. 15, pp. 6776-6787, 2005.

[6] N. M. Sangeetha and U. Maitra, "Supramolecular gels: functions and uses," Chemical Society Reviews, vol. 34, no. 10, pp. 821-836, 2005. 
[7] M. De Loos, B. L. Feringa, and J. H. Van Esch, "Design and application of self-assembled low molecular weight hydrogels," European Journal of Organic Chemistry, no. 17, pp. 3615-3631, 2005.

[8] P. Deindörfer, T. Geiger, D. Schollmeyer, J. H. Ye, and R. Zentel, "Semicarbazides as gel forming agents for common solvents and liquid crystals," Journal of Materials Chemistry, vol. 16, no. 4, pp. 351-358, 2006.

[9] P. Terech and R. G. Weiss, "Low molecular mass gelators of organic liquids and the properties of their gels," Chemical Reviews, vol. 97, no. 8, pp. 3133-3159, 1997.

[10] S. Van der Laan, B. L. Feringa, R. M. Kellogg, and J. Van Esch, "Remarkable polymorphism in gels of new azobenzene bis-urea gelators," Langmuir, vol. 18, no. 19, pp. 7136-7140, 2002.

[11] L. A. Estroff and A. D. Hamilton, "Water gelation by small organic molecules," Chemical Reviews, vol. 104, no. 3, pp. 12011217, 2004.

[12] A. R. Hirst and D. K. Smith, "Two-component gel-phase materials-highly tunable self-assembling systems," Chemistry, vol. 11, no. 19, pp. 5496-5508, 2005.

[13] X. Zhu, P. Duan, L. Zhang, and M. Liu, "Regulation of the chiral twist and supramolecular chirality in co-assemblies of amphiphilic L-glutamic acid with bipyridines," Chemistry, vol. 17, no. 12, pp. 3429-3437, 2011.

[14] P. Duan, L. Qin, X. Zhu, and M. Liu, "Hierarchical self-assembly of amphiphilic peptide dendrons: evolution of diverse chiral nanostructures through hydrogel formation over a wide $\mathrm{pH}$ range," Chemistry, vol. 17, no. 23, pp. 6389-6395, 2011.

[15] T. Jiao, Y. Wang, F. Gao, J. Zhou, and F. Gao, "Photoresponsive organogel and organized nanostructures of cholesterol imide derivatives with azobenzene substituent groups," Progress in Natural Science, vol. 22, no. 1, pp. 64-70, 2012.

[16] T. Jiao, F. Gao, Y. Wang, J. Zhou, F. Gao, and X. Luo, "Supramolecular gel and nanostructures of bolaform and trigonal cholesteryl derivatives with different aromatic spacers," Current Nanoscience, vol. 8, no. 1, pp. 111-116, 2012.

[17] M. de Loos, J. H. van Esch, R. M. Kellogg, and B. L. Feringa, "C3Symmetric, amino acid based organogelators and thickeners: a systematic study of structure-property relations," Tetrahedron, vol. 63, no. 31, pp. 7285-7301, 2007.

[18] P. Dastidar, "Supramolecular gelling agents: can they be designed?" Chemical Society Reviews, vol. 37, no. 12, pp. 26992715, 2008.

[19] M. Suzuki and K. Hanabusa, "L-Lysine-based low-molecularweight gelators," Chemical Society Reviews, vol. 38, no. 4, pp. 967-975, 2009.

[20] P. Mukhopadhyay, Y. Iwashita, M. Shirakawa, S.-I. Kawano, N. Fujita, and S. Shinkai, "Spontaneous colorimetric sensing of the positional isomers of dihydroxynaphthalene in a $1 \mathrm{D}$ organogel matrix," Angewandte Chemie-International Edition, vol. 45, no. 10, pp. 1592-1595, 2006.

[21] T. F. Jiao, Q. Q. Huang, Q. R. Zhang, D. B. Xiao, J. X. Zhou, and F. M. Gao, "Self-assembly of organogels via new luminol imide derivatives: diverse nanostructures and substituent chain effect," Nanoscale Research Letters, vol. 8, no. 278, pp. 1-8, 2013.

[22] Y. Wang, L. Tang, and Y. Wang, "New hydrogen-bonded supramolecular hydrogels and fibers derived from 1,2,4,5benzenetetracarboxylic acid and 4-hydroxypyridine," Chemistry Letters, vol. 35, no. 5, pp. 548-549, 2006.

[23] J. Wu, L. Tang, K. Chen, L. Yan, F. Li, and Y. Wang, "Formation of supramolecular hydrogels with controlled microstructures and stability via molecular assembling in a two-component system," Journal of Colloid and Interface Science, vol. 307, no. 1, pp. 280287, 2007.

[24] J. J. Van Gorp, J. A. J. M. Vekemans, and E. W. Meijer, "C3symmetrical supramolecular architectures: fibers and organic gels from discotic trisamides and trisureas," Journal of the American Chemical Society, vol. 124, no. 49, pp. 14759-14769, 2002.

[25] T. F. Jiao, Y. J. Wang, Q. R. Zhang, J. X. Zhou, and F. M. Gao, "Regulation of substituent groups on morphologies and selfassembly of organogels based on some azobenzene imide derivatives," Nanoscale Research Letters, vol. 8, no. 160, pp. 1-8, 2013.

[26] M. Zinic, F. Vögtle, and F. Fages, "Cholesterol-based gelators," Topics in Current Chemistry, vol. 256, pp. 39-76, 2005. 



Submit your manuscripts at http://www.hindawi.com
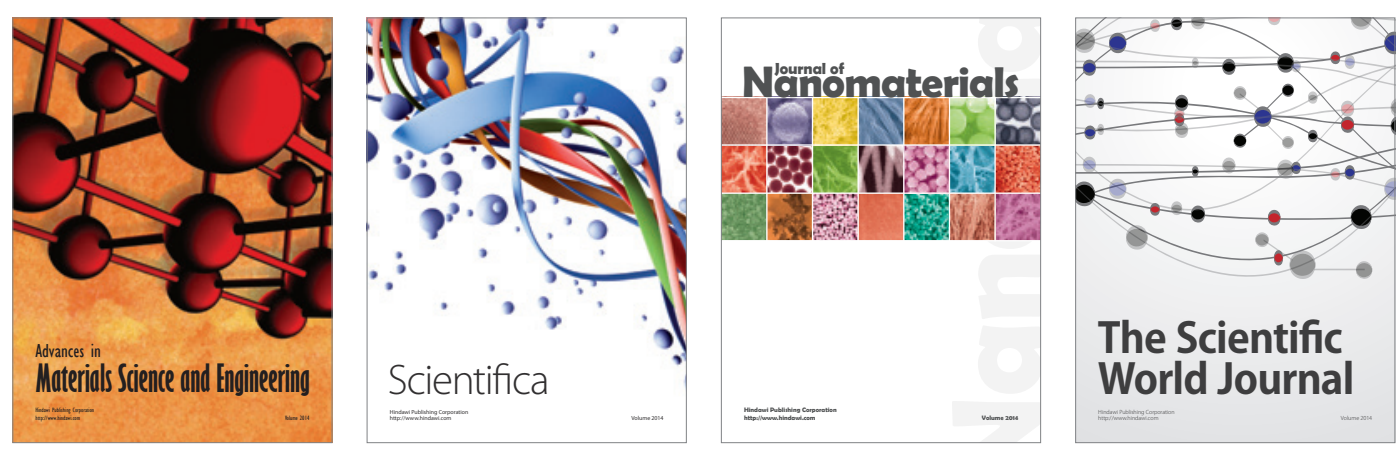

\section{The Scientific World Journal}
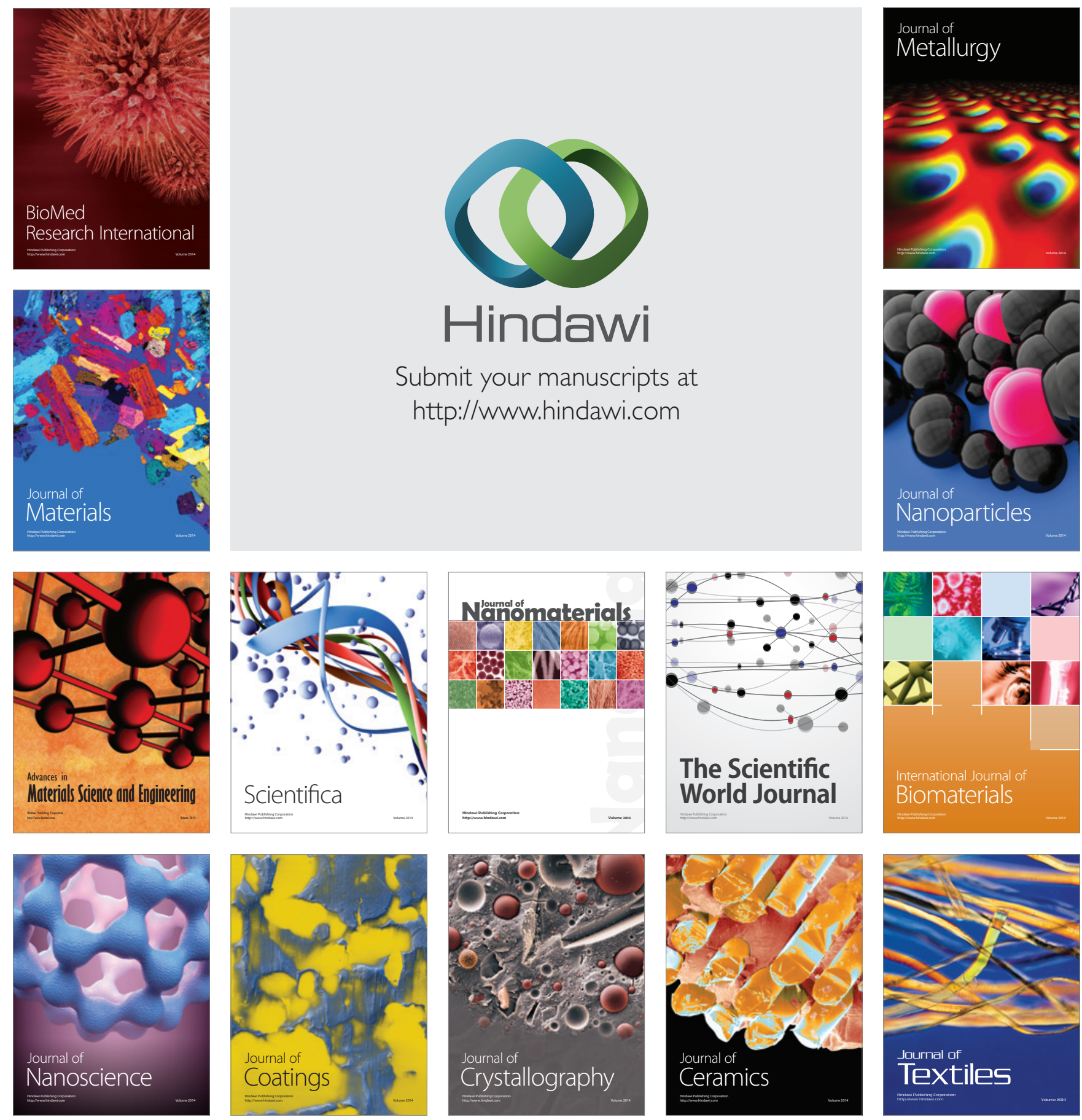